\title{
SAT in Monadic Gödel Logics: a borderline between decidability and undecidability
}

\author{
Matthias Baaz Agata Ciabattoni* Norbert Preining ${ }^{\star \star}$ \\ Technische Universität Wien, Austria
}

\begin{abstract}
We investigate satisfiability in the monadic fragment of firstorder Gödel logics. These are a family of finite- and infinite-valued logics where the sets of truth values $V$ are closed subsets of $[0,1]$ containing 0 and 1 . We identify conditions on the topological type of $V$ that determine the decidability or undecidability of their satisfiability problem.
\end{abstract}

\section{Introduction}

Monadic logic is a first-order logic in which all predicates are unary. Classical monadic logic is a rather simple fragment: decidable (both the validity and satisfiability problem) and having finite model property. The same does not hold for many-valued monadic logics in which a rather complex landscape appears and many questions are still open, see e.g. [3,9,10].

The family of (finite- and infinite-valued) Gödel logics is a prominent example of many-valued logics. Gödel logics, defined in general over sets of truth values $V$ which are closed subsets of $[0,1]$ containing both 0 and 1 , are the only manyvalued logics which are completely specified by the order structure of $V$ [4]. This fact characterizes Gödel logics as logics of comparative truth and renders them an important case of so-called fuzzy logics, see [8].

Different choices of $V$ in general induce different Gödel logics, see [4,6,13]. For $V=\{0,1\}$ the resulting logic coincides with classical logic.

Validity for monadic Gödel logic with $V=[0,1]$ was shown to be undecidable in [2]. Note that in contrast with classical logic, in Gödel logics validity and satisfiability are not dual concepts. A general investigation of the (un)decidability status for the validity problem in monadic Gödel logics was carried out in [3], where it was shown that with the possible exception of Gödel logic $\mathbf{G}_{\uparrow}$ in which $V=\{1-1 / n: n \geq 1\} \cup\{1\}$, validity is undecidable when $V$ is infinite, even when restricted to prenex formulas.

In this paper we investigate the (1-)satisfiability problem $\mathrm{SAT}^{m}$ in monadic Gödel logics. We identify conditions on the topological type of $V$ which characterize Gödel logics with decidable and with undecidable $\mathrm{SAT}^{m}$. SAT ${ }^{m}$ is shown to be decidable for Gödel logics in which 0 is an isolated point in $V$ (i.e., 0 has Cantor-Bendixon rank $|0|_{\mathrm{CB}}=0$, see e.g. [12]). Finite-valued Gödel logics being

* Supported by Vienna Science and Technology Fund (WWTF), project MA07-016.

$\star \star$ Supported by Austrian science foundation (FWF), project P19872-N13 
prominent examples (and any witnessed Gödel logic [11] as well as any prenex Gödel logic can be treated in the same way). In the remaining Gödel logics the presence of at least three predicate symbols, one of which is a constant different from 0 or 1, makes $\mathrm{SAT}^{m}$ undecidable. A complex argument is used to show that without this constant predicate, $\mathrm{SAT}^{m}$ remains undecidable for all Gödel logics in which 0 is a limit point of limit points in $V$ (i.e., $|0|_{\mathrm{CB}} \geq 2$ ). Gödel logic with $V=[0,1]$ being a prominent example. The (un)decidability status of $\mathrm{SAT}^{m}$ is left open for Gödel logics in which $|0|_{\mathrm{CB}}=1$ in $V$ and no constant predicate is available; this case is shown to contain only one logic: Gödel logic $\mathbf{G}_{\downarrow}$ in which $V=\{1 / n: n \in \mathbb{N}\} \cup\{0\}$. SAT ${ }^{m}$ of monadic Gödel logics extended with the projection operator $\triangle$ of [1] is also investigated. This operator allows one to recover classical reasoning inside Gödel logics. We show that the addition of $\triangle$ does not affect the decidability of $\mathrm{SAT}^{m}$ in the finite-valued case, while it does in all infinite-valued Gödel logics (even in the witnessed case) for which $\mathrm{SAT}^{m}$ is undecidable in presence of $\triangle$.

\section{Syntax and Semantics of Gödel Logics}

The language of first-order Gödel logics is identical to that of classical logic.

We call any closed set $V \subseteq[0,1]$ which contains 0 and 1 a Gödel set. Let $V$ be a Gödel set, we denote with $\mathbf{G}_{V}$ the Gödel logic based on the set of truth values $V$. An interpretation (or evaluation) $\varphi_{\mathbf{G}_{V}}$ for $\mathbf{G}_{V}$ maps constants and object variables to elements of a domain $D, n$-ary function symbols to functions from $D^{n}$ into $D$, and $n$-ary predicate symbol $P$ to a function from $D^{n}$ into $V$. (We will use $\varphi_{\mathbf{G}}$ when the truth value set $V$ is clear from the context)

The interpretation $\varphi_{\mathbf{G}_{V}}$ extends in the usual way to a function mapping all terms of the language to an element of the domain. $\varphi_{\mathbf{G}_{V}}$ evaluates atomic formulas $Q \equiv P\left(t_{1}, \ldots, t_{n}\right)$ and the truth constant $\perp$ as

$$
\varphi_{\mathbf{G}_{V}}(Q)=\varphi_{\mathbf{G}_{V}}(P)\left(\varphi_{\mathbf{G}_{V}}\left(t_{1}\right), \ldots, \varphi_{\mathbf{G}_{V}}\left(t_{n}\right)\right) \quad \varphi_{\mathbf{G}_{V}}(\perp)=0
$$

Extension to all formulas is given by

$$
\begin{aligned}
\varphi_{\mathbf{G}_{V}}(A \rightarrow B) & = \begin{cases}1 & \text { if } \varphi_{\mathbf{G}_{V}}(A) \leq \varphi_{\mathbf{G}_{V}}(B) \\
\varphi_{\mathbf{G}_{V}}(B) & \text { otherwise }\end{cases} \\
\varphi_{\mathbf{G}_{V}}(A \wedge B) & =\min \left(\varphi_{\mathbf{G}_{V}}(A), \varphi_{\mathbf{G}_{V}}(B)\right) \\
\varphi_{\mathbf{G}_{V}}(A \vee B) & =\max \left(\varphi_{\mathbf{G}_{V}}(A), \varphi_{\mathbf{G}_{V}}(B)\right)
\end{aligned}
$$

$\neg A, A \leftrightarrow B$ and $A \prec B$ abbreviate $A \rightarrow \perp,(A \rightarrow B) \wedge(B \rightarrow A)$ and $((B \rightarrow$ $A) \rightarrow B)$, respectively; in particular $\varphi_{\mathbf{G}_{V}}(A \prec B)$ is 1 iff either $\varphi_{\mathbf{G}_{V}}(A)<$ $\varphi_{\mathbf{G}_{V}}(B)$, or $\varphi_{\mathbf{G}_{V}}(A)=\varphi_{\mathbf{G}_{V}}(B)=1$. Hence the formula $A \prec B$ expresses 'strictly less' but at 1 (note that $\varphi_{\mathbf{G}_{V}}(1 \prec 1)=1$ ). Let us define the distribution of a formula $A$ and a free variable $x$ with respect to an interpretation $\varphi_{\mathbf{G}_{V}}$ as $\operatorname{Distr}(A(x))=\left\{\varphi_{\mathbf{G}_{V}}^{\prime}(A(x)): \varphi_{\mathbf{G}_{V}}^{\prime} \sim_{x} \varphi_{\mathbf{G}_{V}}\right\}$, where $\varphi_{\mathbf{G}_{V}}^{\prime} \sim_{x} \varphi_{\mathbf{G}_{V}}$ means that $\varphi_{\mathbf{G}_{V}}$ is exactly as $\varphi_{\mathbf{G}_{V}}$ with the possible exception of the domain element 
assigned to $x$. The semantics of quantifiers is given by the infimum and supremum of the corresponding distribution, that is

$$
\varphi_{\mathbf{G}_{V}}(\forall x A(x))=\inf \operatorname{Distr}(A(x)) \quad \varphi_{\mathbf{G}_{V}}(\exists x A(x))=\sup \operatorname{Distr}(A(x)) .
$$

In the case that all infima and suprema are actually realized by an element, i.e., all infima are minima and all suprema are maxima, we speak about witnessed Gödel logics, see e.g. [11].

Definition 1. A formula $A$ is valid or is a tautology (resp. a positive tautology) in $\mathbf{G}_{V}$ if for every interpretation $\varphi_{\mathbf{G}_{V}}, \varphi_{\mathbf{G}_{V}}(A)=1$ (resp. $\left.\varphi_{\mathbf{G}_{V}}(A)>0\right)$. A is 1-satisfiable, or simply satisfiable, if there is an interpretation $\varphi_{\mathbf{G}_{V}}$ such that $\varphi_{\mathbf{G}_{V}}(A)=1$; otherwise $A$ is said to be unsatisfiable.

In contrast with classical logic, in Gödel logics validity and satisfiability are not dual concepts. However the following relation holds: $A$ is a positive tautology if and only if $\neg A$ is unsatisfiable.

Consider the unary operator $\triangle$ with the following meaning [1]:

$$
\varphi_{\mathbf{G}_{V}}(\triangle A)= \begin{cases}1 & \text { if } \varphi_{\mathbf{G}_{V}}(A)=1 \\ 0 & \text { otherwise }\end{cases}
$$

We will investigate monadic Gödel logics (with and without the operator $\triangle$ ) i.e., in which all predicate symbols are (at most) unary and no function symbol occur. Every Gödel set $V$ induces a logic $\mathbf{G}_{V}$ over the language without $\triangle$ and a logic $\mathbf{G}_{V}^{\triangle}$ if $\triangle$ is present. Standard Gödel logic is $\mathbf{G}_{[0,1]}$; i.e., the logic over the full real unit interval as truth value set, see, e.g., $[8,16]$. We use $\mathbf{G}_{n}$ to denote the $n$-valued Gödel logic for $n \geq 2$. Let $G$ be a Gödel logic, we denote with TAUT $\mathrm{T}_{G}^{m}$ and $\mathrm{SAT}_{G}^{m}$ the validity and satisfiability problems of monadic $G$, respectively.

\subsection{Cantor-Bendixon ranks and Gödel sets}

In Gödel logics, the validity of a formula depends only on the relative ordering and the topological type of the truth values of atomic formulas, and not on their specific values. It is therefore important to investigate the topological structure of the underlying truth values sets of Gödel logics, see $[4,13]$. We recall some definitions for the theory of polish spaces, details may be found in [12].

Definition 2. A limit point of a topological space is a point that is not isolated, i.e., for every open neighborhood $U$ of $x$ there is a point $y \subset U$ with $y \neq x$. For any topological space $X$ let $X^{\prime}=\{x \in X: x$ is limit point of $X\}$. We call $X^{\prime}$ the Cantor-Bendixon derivative of $X$ and the operation itself Cantor-Bendixon derivation. Using transfinite recursion we define the iterated Cantor-Bendixon derivatives $X^{\alpha}$, $\alpha$ ordinal, as follows:

$$
X^{0}=X \quad X^{\alpha+1}=\left(X^{\alpha}\right)^{\prime} \quad X^{\lambda}=\bigcap_{\alpha<\lambda} X^{\alpha}, \lambda \text { limit ordinal. }
$$

For any polish space $X$, the least ordinal $\alpha_{0}$ such that $X^{\alpha_{0}}=X^{\alpha}$ for all $\alpha>\alpha_{0}$ is called the Cantor-Bendixon rank of $X$. For any $x \in X$, we define its (CantorBendixon-) rank $|x|_{\mathrm{CB}}=\sup \left\{\alpha: x \in X^{\alpha}\right\}$. 


\section{Decidability Results}

As is well known, the satisfiability problem for monadic classical logic (i.e., $\mathbf{G}_{2}$ ) is decidable, whereas already a single binary predicate symbol leads to undecidability. We show below that this result can be generalized to monadic Gödel logics $\mathbf{G}_{V}$ in which 0 is an isolated point in $V$ (i.e., $|0|_{\mathrm{CB}}=0$ ); satisfiability in these logics, which includes $\mathbf{G}_{n}$, coincides with satisfiability in monadic $\mathbf{G}_{2}$. The same result holds also for any witnessed or prenex monadic Gödel logic. The addition of $\triangle$ to $\mathbf{G}_{n}$ does not change the decidability status of $\mathrm{SAT}_{\mathbf{G}_{n}^{\Delta}}^{m}$, though satisfiability is not anymore equivalent to satisfiability in $\mathbf{G}_{2}$.

Theorem 1. Let $V$ be any Gödel set with $|0|_{\mathrm{CB}}=0 . \mathrm{SAT}_{\mathbf{G}_{V}}^{m}$ is equivalent to $\mathrm{SAT}_{\mathbf{G}_{2}}^{m}$.

Proof. Let $Q$ be any formula of $\mathbf{G}_{V}$. If $Q$ is satisfiable in monadic classical logic then $Q$ is satisfiable in $\mathbf{G}_{V}$. For the converse direction, consider any interpretation $\varphi_{\mathbf{G}}$ of $\mathbf{G}_{V}$ such that $\varphi_{\mathbf{G}}(Q)=1$. An interpretation $\varphi_{\mathbf{C L}}$ of classical logic such that $\varphi_{\mathbf{C L}}(Q)=1$ is defined as follows: for any atomic formula $A$

$$
\varphi_{\mathbf{C L}}(A)= \begin{cases}1 & \text { if } \varphi_{\mathbf{G}}(A)>0 \\ 0 & \text { otherwise }\end{cases}
$$

It is easy to see that for each formula $P,(*) \varphi_{\mathbf{G}}(P)=0$ if and only if $\varphi_{\mathbf{C L}}(P)=0$ and $\varphi_{\mathbf{G}}(P)>0$ if and only if $\varphi_{\mathbf{C L}}(P)=1$. The proof proceeds by induction on the complexity of $P$ and all cases go though for all Gödel logics except when $P$ has the form $\forall x P_{1}(x)$; in this case, being 0 an isolated point in $V, \varphi_{\mathbf{G}}(P)=0$ if and only if there is an element $u$ in the domain of $\varphi_{\mathbf{G}}$ such that $\varphi_{\mathbf{G}}\left(P_{1}(u)\right)=0$; by induction hypothesis $\varphi_{\mathbf{C L}}\left(P_{1}(u)\right)=0$ and hence $\varphi_{\mathbf{C L}}\left(\forall x P_{1}(x)\right)=0$.

Corollary 1. Satisfiability in the existential fragment of all monadic Gödel logics coincides with satisfiability in monadic classical logic.

Corollary 2. Satisfiability in monadic witnessed Gödel logics coincides with satisfiability in monadic classical logic.

Corollary 3. Satisfiability in prenex ${ }^{1}$ monadic Gödel logics coincides with satisfiability in monadic classical logic.

Proof. Let $Q=\mathcal{Q} \bar{x} P$ be any prenex formula, were $\mathcal{Q} \bar{x}$ is the formula prefix and $P$ does not contain quantifiers. The proof is similar to that of Theorem 1 . Assume that $\varphi_{\mathbf{G}}(Q)=1$. As above we can prove $(*)$ for $P \cdot \varphi_{\mathbf{C L}}(Q)=1$ easily follows by induction on the number $n$ of quantifiers in $\mathcal{Q} \bar{x}$.

This result contrasts with the undecidability of the validity problem proved in [3] for all prenex monadic Gödel logics with the exception of $\mathbf{G}_{n}$ and (possibly) $\mathbf{G}_{\uparrow}$.

Corollary 4. $\mathrm{SAT}_{\mathbf{G}_{n}}^{m}$ coincides with $\mathrm{SAT}_{\mathbf{G}_{2}}^{m}$.

\footnotetext{
${ }^{1}$ In general Gödel logics do not admit equivalent prenex formulas, see e.g. [4].
} 
Consider $\mathbf{G}_{n}$ extended with $\triangle$; the formula $\neg \neg A \wedge \neg \triangle A$ is satisfiable in $\mathbf{G}_{n}^{\triangle}$, with $n>2$ (take any interpretation $\varphi_{\mathbf{G}}$ such that $0<\varphi_{\mathbf{G}}(A)<1$ ) but it is not in classical logic. This shows that satisfiability in $\mathbf{G}_{n}^{\triangle}$ is not the same as satisfiability in classical logic. Nevertheless we have

Theorem 2. $\mathrm{SAT}_{\mathbf{G}_{n}^{\Delta}}^{m}$ is decidable.

Proof. Proceed similarly to the decidability proof of $\mathrm{TAUT}_{\mathbf{G}_{n}^{\Delta}}^{m}$ in [3]. Given any monadic formula $P$, it is indeed enough to consider interpretations with domain $\left\{1, \ldots, n^{k}\right\}$, where $k$ is the number of different predicate symbols occurring in $P$. The number of such interpretations is finite (at most $n^{k \cdot n^{k}}$ ).

\section{Undecidability Results}

In this section we prove that $\mathrm{SAT}_{\mathbf{G}_{V}}^{m}$ is undecidable in the following cases: all infinite-valued Gödel logics with $\triangle$ (Section 4.1), all Gödel logics where 0 is not isolated and there are three predicates, one of which is a constant evaluated between 0 and 1 (Section 4.2), and all Gödel logics where 0 has at least CantorBendixon rank 2, i.e., 0 is a limit point of limit points (Section 4.3).

Our results adapt and generalize the undecidability proof of $\mathrm{TAUT}_{\mathbf{G}_{[0,1]}}^{m}$ sketched in [7]. Consider a generic formula $A$ in the classical theory $\mathbf{C E}$ of two equivalence relations $\equiv_{1}$ and $\equiv_{2}$. Without loss of generality we can assume that $A$ is in prenex and disjunctive normal form, i.e., $A$ is of the form:

$$
A=\mathcal{Q}^{*} \bigvee_{j} \bigwedge_{k}\left(x_{j}^{k} \equiv_{i} y_{j}^{k}\right)^{l}
$$

where $\mathcal{Q}^{*}$ is the formula prefix and $l$ is either -1 , in which case the atomic equivalence is negated, or 1 , in which case it is positive (not negated).

In the subsections below we will translate $A$ into formulas of $\mathbf{G}_{V}$ by replacing each term $\left(x_{j}^{k} \equiv_{i} y_{j}^{k}\right)^{l}$ in $A$ by suitable $\sigma\left(\left(u_{j}^{k} \equiv_{i} v_{j}^{k}\right)^{l}\right)$. As notational extension we write $\sigma(A)=\mathcal{Q}^{*} \bigvee_{j} \bigwedge_{k} \sigma\left(\left(x_{j}^{k} \equiv_{i} y_{j}^{k}\right)^{l}\right)$.

Lemma 1. Given two interpretations $\varphi_{\mathbf{C E}}$ and $\varphi_{\mathbf{G}}$. If for each term $u_{j}^{k}$ and $v_{j}^{k}$

$$
\begin{gathered}
\varphi_{\mathbf{C E}}\left(\left(u_{j}^{k} \equiv_{i} v_{j}^{k}\right)^{l}\right)=1 \quad \Leftrightarrow \quad \varphi_{\mathbf{G}}\left(\sigma\left(u_{j}^{k} \equiv_{i} v_{j}^{k}\right)^{l}\right)=1 \\
(\text { with } l=-1,1) \text { and } \varphi_{\mathbf{G}}\left(\sigma\left(u_{j}^{k} \equiv_{i} v_{j}^{k}\right)^{l}\right)=1 \text { or } \varphi_{\mathbf{G}}\left(\sigma\left(u_{j}^{k} \equiv_{i} v_{j}^{k}\right)^{l}\right) \leq k<1 \text { then } \\
\varphi_{\mathbf{C E}}(A)=1 \quad \Leftrightarrow \quad \varphi_{\mathbf{G}}(\sigma(A))=1 .
\end{gathered}
$$

\subsection{Infinite-valued Gödel logics with $\triangle$}

We show the undecidability of $\mathrm{SAT}_{\mathbf{G}_{V}^{\Delta}}$, where $V$ is any infinite Gödel set. Our argument, which applies also to all infinite-valued witnessed Gödel logics with $\triangle$, is similar to that for the undecidability of $\mathrm{TAUT}_{\mathbf{G}_{V}}$ in [3]. 
Theorem 3. Let $V$ be any infinite Gödel set. Satisfiability of monadic formulas in $\mathbf{G}_{V}^{\triangle}$, with at least two predicate symbols, is undecidable.

Proof. Validity of formulas in the classical theory $\mathbf{C E}$ of two equivalence relations was shown in [15] to be r.e. but not recursive. As a formula in classical logic is valid if and only if its negation is unsatisfiable, the satisfiability problem in $\mathbf{C E}$ is undecidable and not even recursively enumerable. The theorem's claim follows by faithfully interpret $\mathbf{C E}$ in the monadic fragment of $\mathbf{G}_{V}^{\triangle}$. Indeed, let $P_{1}$ and $P_{2}$ be different unary predicate symbols in $\mathbf{G}_{V}^{\triangle}$. A translation $\sigma(A)$ of CE-formulas $A$ into monadic $\mathbf{G}_{V}^{\triangle}$-formulas is simply defined by translating each atomic formula in $A$ as follows: $\sigma\left(x_{j}^{k} \equiv_{i} y_{j}^{k}\right)=\triangle\left(P_{i} x \leftrightarrow P_{i} y\right)$.

Assume first that a $\mathbf{C E}$-valuation $\varphi_{\mathbf{C E}}$ of $A$ is given such that $\varphi_{\mathbf{C E}}(A)=1$. By the Löwenheim-Skolem's theorem we can assume the universe $U$ of its model to be countable, without loss of generality. Therefore also the set of equivalence classes $[u]_{i}=\left\{v \mid \varphi_{\mathbf{C E}}\left(u \equiv_{i} v\right)=1\right\}$ with respect to the two equivalence relations is countable. Since our Gödel set $V$ is infinite, there exists a injection $\lambda$ :

$$
\lambda:\left\{[u]_{1},[u]_{2}: u \in U\right\} \rightarrow V \backslash\{0,1\} .
$$

Using this injection we define the valuation in $\mathbf{G}_{V}^{\triangle}$ as

$$
\varphi_{\mathbf{G}}\left(P_{i} u\right)=\lambda\left([u]_{i}\right) .
$$

We now show that the assumptions of Lemma 1 are fulfilled:

$$
\begin{aligned}
& \varphi_{\mathbf{C E}}\left(u_{j}^{k} \equiv_{i} v_{j}^{k}\right)=1 \quad \Leftrightarrow \quad\left[u_{j}^{k}\right]_{i}=\left[v_{j}^{k}\right]_{i} \quad \Leftrightarrow \quad \lambda\left(\left[u_{j}^{k}\right]_{i}\right)=\lambda\left(\left[v_{j}^{k}\right]_{i}\right) \\
& \Leftrightarrow \quad \varphi_{\mathbf{G}}\left(P_{i} u_{j}^{k}\right)=\varphi_{\mathbf{G}}\left(P_{i} u_{j}^{k}\right) \quad \Leftrightarrow \quad \varphi_{\mathbf{G}}\left(P_{i} u_{j}^{k} \leftrightarrow P_{i} u_{j}^{k}\right)=1 \\
& \Leftrightarrow \quad \varphi_{\mathbf{G}}\left(\triangle\left(P_{i} u_{j}^{k} \leftrightarrow P_{i} u_{j}^{k}\right)\right)=1 \quad \Leftrightarrow \quad \varphi_{\mathbf{G}}\left(\sigma\left(u_{j}^{k} \equiv_{i} v_{j}^{k}\right)\right)=1
\end{aligned}
$$

We can similary prove that $\varphi_{\mathbf{C E}}\left(u_{j}^{k} \not_{i} v_{j}^{k}\right)=1 \quad \Leftrightarrow \quad \varphi_{\mathbf{G}}\left(\sigma\left(u_{j}^{k} \not_{i} v_{j}^{k}\right)\right)=1$. Moreover, $\varphi_{\mathbf{G}}\left(\sigma\left(u_{j}^{k} \equiv_{i} v_{j}^{k}\right)\right)$ and $\varphi_{\mathbf{G}}\left(\sigma\left(u_{j}^{k} \not_{i} v_{j}^{k}\right)\right)$ are either 0 or 1 . Hence, by Lemma $1, \varphi_{\mathbf{G}}(\sigma(A))=1$.

Now assume that there exists a valuation $\varphi_{\mathbf{G}}$ in $\mathbf{G}_{V}^{\triangle}$ such that $\varphi_{\mathbf{G}}(\sigma(A))=1$. Define the valuation $\varphi_{\mathbf{C E}}$ in classical logic as

$$
\varphi_{\mathbf{C E}}\left(u \equiv_{i} v\right)=1 \text { iff } \varphi_{\mathbf{G}}\left(\sigma\left(u \equiv_{i} v\right)\right)=1
$$

the above equivalence chains together with Lemma 1 give $\varphi_{\mathbf{C E}}(A)=1$.

Corollary 5. SAT ${ }^{m}$ is undecidable in prenex or witnessed Gödel logics with $\triangle$, in presence of two predicate symbols.

Proof. The very same proof as for Theorem 3 can be used.

The undecidability result applies also to the monadic fragments of Eukasiewicz and product logics, two important formalizations of fuzzy logic [8]. For the former logic, Ragaz [14] proved the undecidability of the satisfiability problem in presence of at least four predicate symbols. This result was extended in [5] to product logic. 
Corollary 6. Satisfiability of monadic Lukasiewicz and product logic extended with $\triangle$ is undecidable, in presence of at least two predicate symbols.

Proof. Follows by the embeddability of $\mathbf{G}_{[0,1]}^{\triangle}$ in Lukasiewicz and product logic extended with $\triangle$, cf. [5].

\subsection{Infinite Gödel sets with $|0|_{\mathrm{CB}}>0$}

We show the undecidability of $\operatorname{SAT}_{\mathbf{G}_{V}}^{m}$ where $V$ is infinite, the truth value 0 has Cantor-Bendixon rank $|0|_{\mathrm{CB}}>0$ in $V$ (i.e., 0 is not isolated) and there is a (0-ry) predicate $S$ which is always evaluated to a real between 0 and 1 .

Theorem 4. $\mathrm{SAT}_{\mathbf{G}_{V}}^{m}$ is undecidable in any Gödel logic $\mathbf{G}_{V}$ where $|0|_{\mathrm{CB}}>0$ in presence of at least three predicates one of which is a constant $S$ such that $0<\varphi_{\mathbf{G}}(S)<1$ for each valuation $\varphi_{\mathbf{G}}$.

Proof. Proceed similarly to that of Theorem 3. Here we define the (local) translation $\sigma$ as follows:

$$
\begin{aligned}
\sigma\left(x_{j}^{k} \equiv_{i} y_{j}^{k}\right) & =\left(P_{i} x_{j}^{k} \leftrightarrow P_{i} y_{j}^{k}\right) \\
\sigma\left(x_{j}^{k} \not_{i} y_{j}^{k}\right) & =\left(P_{i} x_{j}^{k} \leftrightarrow P_{i} y_{j}^{k}\right) \rightarrow S
\end{aligned}
$$

and translate the $\mathbf{C E}$ formula $A$ as $\tau(A)=\sigma(A) \wedge \forall x\left(P_{1} x \prec S\right) \wedge \forall x\left(P_{2} x \prec S\right)$.

Assume first that a valuation $\varphi_{\mathrm{CE}}$ of $A$ in classical logic is given such that $\varphi_{\mathbf{C E}}(A)=1$. Following the first steps of the proof of Theorem 3 we define

$$
\varphi_{\mathbf{G}}\left(P_{i} u\right)=\lambda\left([u]_{i}\right)
$$

but here the injection $\lambda$ maps equivalence classes into truth values below $\varphi_{\mathbf{G}}(S)$ :

$$
\lambda:\left\{[u]_{i},[u]_{2}: u \in U\right\} \rightarrow V \cap\left(0, \varphi_{\mathbf{G}}(S)\right) .
$$

This can always be achieved as 0 is not isolated, thus, below any given point in $V$, in our case below $\varphi_{\mathbf{G}}(S)$, there are at least countably many truth values.

We now show that the remaining assumptions of Lemma 1 are fulfilled (by definition $\varphi_{\mathbf{G}}\left(\sigma\left(u_{j}^{k} \equiv_{i} v_{j}^{k}\right)\right)$ and $\varphi_{\mathbf{G}}\left(\sigma\left(u_{j}^{k} \not_{i} v_{j}^{k}\right)\right)$ are either 1 or below $\left.\varphi_{\mathbf{G}}(S)\right)$. Obviously $\varphi_{\mathbf{C E}}\left(u_{j}^{k} \equiv_{i} v_{j}^{k}\right)=1$ iff $\varphi_{\mathbf{G}}\left(P_{i} u_{j}^{k}\right)=\varphi_{\mathbf{G}}\left(P_{i} v_{j}^{k}\right)$. Moreover

$$
\begin{gathered}
\varphi_{\mathbf{C E}}\left(u_{j}^{k} \not_{i} v_{j}^{k}\right)=1 \quad \Leftrightarrow \quad\left[u_{j}^{k}\right]_{i} \neq\left[v_{j}^{k}\right]_{i} \quad \Leftrightarrow^{1} \quad \lambda\left(\left[u_{j}^{k}\right]_{i}\right) \neq \lambda\left(\left[v_{j}^{k}\right]_{i}\right) \\
\varphi_{\mathbf{G}}\left(P_{i} u_{j}^{k}\right) \neq \varphi_{\mathbf{G}}\left(P_{i} u_{j}^{k}\right) \Leftrightarrow \quad \Leftrightarrow \quad \varphi_{\mathbf{G}}\left(P_{i} u_{j}^{k} \leftrightarrow P_{i} u_{j}^{k}\right) \leq \varphi_{\mathbf{G}}(S) \\
\left.\Leftrightarrow \quad \varphi_{\mathbf{G}}\left(\left(P_{i} u_{j}^{k} \leftrightarrow P_{i} u_{j}^{k}\right) \rightarrow S\right)\right)=1 \quad \Leftrightarrow \quad \varphi_{\mathbf{G}}\left(\sigma\left(u_{j}^{k} \not_{i} v_{j}^{k}\right)\right)=1
\end{gathered}
$$

(where ${ }^{1}$ follows from the injectivity of $\lambda$ ). By Lemma 1 , we have $\varphi_{\mathbf{G}}(\sigma(A))=1$ and by the definition of $\varphi_{\mathbf{G}}, \varphi_{\mathbf{G}}(\tau(A))=1$.

For the converse direction, assume to have a valuation $\varphi_{\mathbf{G}}$ such that $\varphi_{\mathbf{G}}(\tau(A))=$ 1. Define the valuation $\varphi_{\mathbf{C E}}$ in a straightforward way as

$$
\varphi_{\mathbf{C E}}\left(u \equiv_{i} v\right)=1 \text { iff } \varphi_{\mathbf{G}}\left(\sigma\left(u \equiv_{i} v\right)\right)=1 .
$$

Notice that by $\varphi_{\mathbf{G}}\left(\forall x\left(P_{i} x \prec S\right)\right)=1$ follows that $\varphi_{\mathbf{G}}\left(P_{i} x\right)<\varphi_{\mathbf{G}}(S)<1$ for all $x$. The equivalences above together with Lemma 1 give $\varphi_{\mathbf{C E}}(A)=1$. 


\subsection{Infinite Gödel sets with $|0|_{\mathrm{CB}} \geq 2$}

We show the undecidability of $\mathrm{SAT}_{\mathbf{G}_{V}}^{m}$ where $V$ is infinite, 0 has Cantor-Bendixon rank $|0|_{\mathrm{CB}} \geq 2$ (i.e., 0 is limit point of limit points) and the language of $\mathbf{G}_{V}$ contains at least three predicate symbols. $\mathbf{G}_{[0,1]}$ being a prominent example.

Theorem 5. $\mathrm{SAT}_{\mathbf{G}_{V}}^{m}$ is undecidable when $|0|_{\mathrm{CB}} \geq 2$ in $V$ and in presence of at least three predicate symbols.

Proof. Proceed similarly to the proofs of Theorem 4. First notice that the central property used in this proof (cf. Lemma 1) is that there is a way to decide whether an evaluation is below 1 or it is 1 . Indeed, for the reverse direction of the undecidability proof (i.e., given a satisfying Gödel interpretation $\varphi_{\mathbf{G}_{V}}$ we have to construct a satisfying interpretation in $\mathbf{C E}$ ) we need to select an open interval in $V$ strictly between 0 and 1, objects with valuations of the equivalence predicates $P_{i}$ within that interval, and use them to define the equivalence classes in CE. In Theorem 4, the predicate constant is used to this purpose. Here a formula $\exists y \exists z\left(P_{i} z \prec P_{i} y \wedge \ldots\right)$ would not be enough as our 'strictly less' relation $\prec$ collapses at 1 (see Section 2 ) and therefore we cannot be sure that $\varphi_{\mathbf{G}_{V}}\left(P_{i} y\right)$ and $\varphi_{\mathbf{G}_{V}}\left(P_{i} z\right)$ are choosen below 1 . To overcome this problem we use the third predicate $P$ whose valuation we force to be a decreasing sequence to 0 . This can be expressed by $\neg \forall x P x \wedge \forall x \neg \neg P x$, as the first conjunct says that the infimum of all evaluations of $P$ is 0 , and the second that every valuation of $P$ is bigger than 0 . So we can say that either the valuation of $P x$ is 1 , or we can find $y$ and $z$ 'below' $x$ (i.e., the valuations of $P y$ and $P z$ are below the valuation of $P x)$ defining the needed open interval. This leads to the formula $\forall x(P x \vee \exists y \exists z(P z \prec P y \wedge P y \prec P x))$; on the other hand requiring the existence of objects $w$ with $\varphi_{\mathbf{G}_{V}}\left(P_{i} w\right)$ within the interval can be simply expressed by $\exists w\left(P z \prec P_{1} w \prec P y \wedge P z \prec P_{2} w \prec P y\right)$.

Consider now the forward direction of the proof, i.e., constructing a satisfying valuation in $\mathbf{G}_{V}$ from a satisfying valuation in $\mathbf{C E}$. The use of the formulas above complicates the matters as we have to deal with countably many $\varphi_{\mathbf{G}}(P x)$ for $x \in U_{\mathbf{G}}$ and below each of them open intervals (coming from $P y$ and $P z$ ), in which the equivalence classes are interpreted in parallel. This gives rise to the condition that the Cantor-Bendixon rank of 0 is at least 2. This 'parallel construction' also hints that in translating our formula $A$ of $\mathbf{C E}$ into a formula $\tau(A)$ of $\mathbf{G}_{V}$ we will duplicate the universe $U_{\mathbf{C E}}$ for each interval so that we can faithfully embed the equivalence classes of $\mathbf{C E}$ into the respective intervals. This leads to the fact that in the translated formula $\tau(A)$ the quantifiers act over a much larger universe, as we have to duplicate the universe for each interval. To confine the valuations of elements of the 'correct' interval we add in the formula (1a) below disjuncts evaluating to 1 for objects outside the considered interval.

We are now ready to present the formal definition of the translation of our formula $A$ in $\mathbf{C E}$ into $\mathbf{G}_{V}$ which uses the (not anymore local) translation $\sigma_{a, b}(A)$, 
where $a$ and $b$ define the interval $[b, a]$ in which the evaluation takes place:

$$
\begin{aligned}
& \sigma_{a, b}(\forall r B)=\forall r\left(P_{1} r \prec P b \vee P a \prec P_{1} r \vee P_{2} r \prec P b \vee P a \prec P_{2} r \vee \sigma_{a, b}(B)\right) \\
& \sigma_{a, b}(\exists r B)=\exists r\left(\left(P b \prec P_{1} r \prec P a\right) \wedge\left(P b \prec P_{2} r \prec P a\right) \wedge \sigma_{a, b}(B)\right) \\
& \sigma_{a, b}\left(\bigvee_{j} \bigwedge_{k}\left(r_{j}^{k} \equiv_{i} s_{j}^{k}\right)^{l}\right)=\bigvee_{j} \bigwedge_{k} \sigma\left(\left(r_{j}^{k} \equiv_{i} s_{j}^{k}\right)^{l}\right) \\
& \sigma_{a, b}\left(r \equiv_{i} s\right)=\left(P_{i} r \leftrightarrow P_{i} s\right) \\
& \left.\sigma_{a, b}\left(r \not_{i} s\right)=\left(\left(P_{i} r \leftrightarrow P_{i} s\right) \rightarrow P a\right)\right)
\end{aligned}
$$

The translation $\tau(A)$ of the $\mathbf{C E}$ formula $A$ is defined as:

$$
\begin{aligned}
& \tau(A)=\neg \forall x P x \wedge \forall x \neg \neg P x \wedge \\
& \forall x(P x \vee \exists y \exists z[ \\
& P z \prec P y \wedge P y \prec P x \wedge \\
& \forall u(P u \rightarrow P z \vee P y \rightarrow P u) \wedge \\
& \exists w\left(P z \prec P_{1} w \prec P y \wedge P z \prec P_{2} w \prec P y\right) \wedge \\
&\left.\left.\sigma_{y, z}(A)\right]\right)
\end{aligned}
$$

As in the proofs of Theorems 3 and 4 we assume first that $\varphi_{\mathbf{C E}}(A)=1$ and we construct an interpretation $\varphi_{\mathbf{G}}$ of $\mathbf{G}_{V}$ in which $\varphi_{\mathbf{G}}(\tau(A))=1$. By the Löwenheim-Skolem's theorem we assume that the domain $U_{\mathbf{C E}}$ of $\varphi_{\mathbf{C E}}$ is countable. As mentioned above we duplicate the universe $U_{\mathbf{C E}}$ countably many times, and for good measure we throw in another countable set of objects $\left(c_{n}\right)$ which we use to define the decreasing sequence $P c_{n}$ to 0 . Thus, the universe of our valuation $\varphi_{\mathbf{G}}$ is defined as $U_{\mathbf{G}}=\left\{u^{n}: u \in U_{\mathbf{C E}}, n \in \mathbb{N}\right\} \cup\left\{c_{n}: n \in \mathbb{N}\right\}$, where all the $u^{n}$ and $c_{n}$ are different. Note that we add the index $n$ as superscript to $u$ to indicate copies of the elements $u \in U_{\mathbf{C E}}$.

The values of $P$ under $\varphi_{\mathbf{G}}$ meet the following requirements: $\varphi_{\mathbf{G}}\left(P c_{n}\right)$ satisfies (i) $\varphi_{\mathbf{G}}\left(P c_{n+1}\right)<\varphi_{\mathbf{G}}\left(P c_{n}\right)$, (ii) $\lim _{n \rightarrow \infty} \varphi_{\mathbf{G}}\left(P c_{n}\right)=0$, (iii) $\varphi_{\mathbf{G}}\left(P c_{n}\right) \in V^{\prime} \backslash$ $\{0,1\}$, where $V^{\prime}$ is the Cantor-Bendixon derivative of $V$, and (iv) $\varphi_{\mathbf{G}}\left(P u^{n}\right)=1$ for all $n$ and $u \in U_{\mathbf{C E}}$. This definition makes sure that $\varphi_{\mathbf{G}}((2 \mathrm{a}))=1$.

From $|0|_{\mathrm{CB}} \geq 2$ and (iii) follow that below any given $\varphi_{\mathbf{G}}\left(P c_{n}\right)$ there exist countably many disjoint open intervals, each containing countably many truth values. Define $f: \mathbb{N} \rightarrow \mathbb{N}$ and $K_{n}$ such that: (a) $f$ is strictly monotone increasing, and (b) the open interval $K_{n}=\left(\varphi_{\mathbf{G}}\left(P c_{f(n)+1}\right), \varphi_{\mathbf{G}}\left(P c_{f(n)}\right)\right)$ contains countably many truth values. As a consequence of (a) and (b) we have (c) the intervals $K_{n}$ are all disjoint.

Since there are at least countably many truth values in each $K_{n}$ there is for any $n$ an injection $\lambda_{n}:\left\{[u]_{1},[u]_{2}: u \in U_{\mathbf{C E}}\right\} \rightarrow K_{n} \cap V$. The valuation of $P_{i}\left(u^{n}\right)$ is then defined as

$$
\varphi_{\mathbf{G}}\left(P_{i}\left(u^{n}\right)\right)=\lambda_{n}\left([u]_{i}\right) .
$$

This ensures that $(2 \mathrm{e})$ is satisfied. To complete the definition of the valuation of atomic formulas we set $\varphi_{\mathbf{G}}\left(P_{i}\left(c_{n}\right)\right)=1$ for all $n$. 
We will now show that $\varphi_{\mathbf{G}}(\tau(A))=1$. Consider the universal quantifier $\forall x$ in (2b) and pick up an arbitrary element $x$ from $U_{\mathbf{G}}$. If $x=u^{n}$ then $\varphi_{\mathbf{G}}(P x)=1$ (see (iv) above). Assume now that $x=c_{n}$. We choose $c_{f(n)}$ for $y$ and $c_{f(n)+1}$ for $z$. (Remember that the interval $K_{n}$ defined through the evaluations of $P$ with these elements contains countably many truth values). It is easy to see that with these chosen $y$ and $z$ the parts $(2 \mathrm{c})$ and $(2 \mathrm{~d})$ are satisfied.

To prove that $\varphi_{\mathbf{G}}(\tau(A))=1$ it remains to show that $\varphi_{\mathbf{G}}\left(\sigma_{n}(A)\right)=1$ where $\sigma_{n}$ is a shorthand for $\sigma_{c_{f(n)}, c_{f(n)+1}}$. We can indeed give a selection function for the existentially quantified variables: If all the quantifiers in front of an existential quantifier are instantiated, simply drop all the super-scripts in $u^{n}$, consider the resulting assignment in $\mathbf{C E}$, and use the object selected by the existential quantifier there, adding the index of the current interval in which we evaluate. Therefore the existential quantifiers are always evaluated in the current interval, and thus the first two conjuncts of (1b) are satisfied.

On the other hand considering the universal quantifier and (1a) we see that if the object instantiating the universal quantifier is outside the current interval, i.e., the valuations are outside the interval defined by $\varphi_{\mathbf{G}}(P y)=\varphi_{\mathbf{G}}\left(P c_{f(n)}\right)$ and $\varphi_{\mathbf{G}}(P z)=\varphi_{\mathbf{G}}\left(P c_{f(n)+1}\right)$, the evaluation of (1a) immediately becomes 1 .

So we can assume in the following that all objects instantiating quantified variables in (2f), i.e., in $\sigma_{y, z}(A)$, give valuations of $P_{1}$ and $P_{2}$ within the interval under discussion. The very same computations as in Theorem 4 (with $P c_{f(n)}$ playing the role of $S$ ) show $\varphi_{\mathbf{G}}\left(\sigma_{n}(A)\right)=1$ for each $n$ if $\varphi_{\mathbf{C E}}(A)=1$. Hence $\varphi_{\mathbf{G}}(\tau(A))=1$ if $\varphi_{\mathbf{C E}}(A)=1$.

For the reverse direction assume that $\varphi_{\mathbf{G}}(\tau(A))=1$. For $\varphi_{\mathbf{G}}$ the following hold: (i) there exists an $u^{\prime}$ such that $0<\varphi_{\mathbf{G}}\left(P u^{\prime}\right)<1$, as $\varphi_{\mathbf{G}}((2 \mathrm{a}))=1$; (ii) there are $y=v$ and $z=w$ such that (2c)-(2e) hold. Define the universe of $\varphi_{\mathbf{C E}}$ as

$$
U_{\mathbf{C E}}=\left\{u \in U_{\mathbf{G}}: \varphi_{\mathbf{G}}(P w)<\varphi_{\mathbf{G}}\left(P_{i} u\right)<\varphi_{\mathbf{G}}(P v), i=1,2\right\}
$$

Note that $U_{\mathbf{C E}}$ cannot be empty as $\varphi_{\mathbf{G}}((2 \mathrm{e}))=1$. Define a valuation $\varphi_{\mathbf{C E}}$ as

$$
\varphi_{\mathbf{C E}}\left(a \equiv_{i} b\right)=1 \quad \text { iff } \quad \varphi_{\mathbf{G}}\left(P_{i} a \leftrightarrow P_{i} b\right)=1
$$

from which follows that $\varphi_{\mathbf{C E}}(A)=1$ being $\varphi_{\mathbf{C E}}(A)$ nothing but the valuation $\varphi_{\mathbf{G}}\left(\sigma_{v, w}(A)\right)$.

Notice that all infinite-valued Gödel $\operatorname{logics} \mathbf{G}_{V}$ with at least three predicate symbols satisfy the hypothesis of the theorem above, with the exception of those in which $|0|_{\mathrm{CB}}=0$ or $|0|_{\mathrm{CB}}=1$ in $V$. In the former case, Theorem 1 ensures the decidability of $\mathrm{SAT}_{\mathbf{G}_{V}}^{m}$. We show below that the latter case, which is the only case left open, refers, in fact, to one Gödel logic: the one known as $\mathbf{G}_{\downarrow}$ and in which $V=\{1 / n: n \in \mathbb{N}\} \cup\{0\}$.

Proposition 1. If $V$ has $|0|_{\mathrm{CB}}=1$, then $\mathrm{SAT}_{\mathbf{G}_{V}}$ is equivalent to $\mathrm{SAT}_{\mathbf{G}_{\downarrow}}$.

Proof. For any Gödel set $V$ in which $|0|_{\mathrm{CB}}=1$ there is a $\lambda \notin V, 0<\lambda<1$, such that below $\lambda$ there are only isolated truth values and 0 . Using the same technique as in Theorem 1 but projecting everything above $\lambda$ to 1 we see that all such $V$ are order isomorphic to $\{1 / n: n \in \mathbb{N}\} \cup\{0\}$; i.e., to the truth values set of $\mathbf{G}_{\downarrow}$. 


\section{References}

1. M. Baaz. Infinite-valued Gödel logics with 0-1-projections and relativizations. In Proceedings Gödel 96. Kurt Gödel's Legacy. LNL 6, Springer, 23-33. 1996.

2. M. Baaz, A. Ciabattoni and C.G. Fermüller. Herbrand's Theorem for Prenex Gödel Logic and its Consequences for Theorem Proving. Proceedings of LPAR'2001. LNAI 2250, Springer, 201-216. 2001.

3. M. Baaz, A. Ciabattoni and C.G. Fermüller. Monadic Fragments of Gödel Logics: Decidability and Undecidability Results. Proceedings of LPAR'200\%. LNAI 4790, Springer, 77-91. 2007.

4. M. Baaz, N. Preining, R. Zach. First-order Gödel logics. Annals of Pure and Applied Logic, 147:23-47, 2007.

5. M. Baaz, P. Hájek, D. Svejda and J. Krajícek. Embedding Logics into Product Logic. Studia Logica, vol. 61(1), pp. 35-47. 1998.

6. A. Beckmann, M. Goldstern, and N. Preining. Continuous Fraïssé conjecture. Order, 25(4):281-298, 2008.

7. D.M. Gabbay. Decidability of some intuitionistic predicate theories. J. of Symbolic Logic, vol. 37, pp. 579-587. 1972.

8. P. Hájek. Metamathematics of Fuzzy Logic. Kluwer, 1998.

9. P. Hájek. Arithmetical complexity of fuzzy predicate logics: a survey II. Annals of Pure and Applied Logic. To appear.

10. P. Hájek. Monadic Fuzzy Predicate Logics. Studia Logica, 71(2), pp. 165-175. 2002.

11. P. Hájek and P. Cintula. On theories and models in fuzzy predicate logics. Journal of Symbolic Logic, 71(3), pp. 863-880, 2006.

12. A. S. Kechris. Classical Descriptive Set Theory. Springer, 1995.

13. N. Preining. Complete Recursive Axiomatizability of Gödel Logics. PhD thesis, Vienna University of Technology, Austria, 2003.

14. M. Ragaz. Die Unentscheidbarkeit der einstelligen unendlichwertigen Prädikatenlogik. Arch. math. Logik, 23 (1983), 129-139.

15. H. Rogers. Certain logical reduction and decision problems. Annals of Mathematics, vol. 64, pp. 264-284. 1956.

16. G. Takeuti, T. Titani. Intuitionistic fuzzy logic and intuitionistic fuzzy set theory. J. of Symbolic Logic, 49 (1984), 851-866. 